\title{
Characterization of the anamorphic and spatial frequency dependent phenomenon in Liquid Crystal on silicon displays
}

L. Lobato

laura.lobato.87@gmail.com

\section{A. Lizana}

\section{A. Márquez}

\section{Moreno}

C. Iemmi

J. Campos
Departamento de Física, Universidad Autónoma de Barcelona, 08193 Bellaterra, Spain

Departamento de Física, Universidad Autónoma de Barcelona, 08193 Bellaterra, Spain

Dept. de Física, Ingeniería de Sistemas y Teoría de la Señal, Universidad de Alicante, Ap. 99, 03080 Alicante, Spain

Dept. de Ciencia de Materiales, Óptica y Tecnología Electrónica, Universidad Miguel Hernández, Elche, Spain

Dept. de Física, Fac. de Ciencias Exactas y Naturales, Universidad de Buenos Aires, 1428 Buenos Aires, Argentina

Departamento de Física, Universidad Autónoma de Barcelona, 08193 Bellaterra, Spain

The diffractive efficiency of Liquid Crystal on Silicon (LCOS) displays can be greatly diminished by the appearance of temporal phase fluctuations in the reflected beam, depolarization effects and also because of phase modulation depths smaller than $2 \pi$. In order to maximize the efficiency of the Diffractive Optical Elements (DOEs) implemented in the LCoS device, the Minimum Euclidean Distance principle can be applied. However, not all the diffractive elements can be corrected in the same way due to the anamorphic and spatial frequency dependent phenomenon, which is related to the LCOS response, largely depending on the period and the spatial orientation of the generated DOE. Experimental evidence for the anamorphic and spatial frequency dependent phenomenon is provided in this paper, as well as a comparative study between the LCOS display response for binary gratings of different periods. [D0I: 10.2971/jeos.2011.11012S]

Keywords: LCOS device, anamorphic phenomenon, diffractive optical element, global retardance, diffractive efficiency

\section{INTRODUCTION}

Liquid Crystal on Silicon (LCoS) displays are a subtype of Liquid Crystal Displays (LCDs) working by reflection. Some of the most noticeable features of these devices are their very high light efficiency and resolution, and also their great capability to spatially modulate light beams [1], this last being especially important in applications requiring phase-shift modulation of the incident wavefront. Consequently, they have attained widespread use in applications such as diffractive optics [2], adaptive optics [3], optical metrology [4], polarimetry [5] or medical physics [6]. In this framework, complete LCoS characterization and optimization becomes essential to achieve better performance of these devices and thus providing greater efficiencies in diffractive optics.

While most commercial LCDs do not offer the chance to access the parameters defining the electro-optic modulation properties of the LC device, the Parallel Aligned (PA) LCoS used in this study allows different electrical sequences formats to be addressed, which may also result in different responses and efficiencies [7, 8]. Modern LCoS displays are digitally addressed, and the electrical sequences are based on pulse-width modulation scheme. The modulated pulses can lead to flicker in the optical beam because of the limited viscosity of the LC molecules. This may introduce undesirable temporal phase fluctuations $[9,10]$ and/or a certain amount of depolarization $[11,12]$ on the light beam reflected by the LCoS display. Because these phenomena have a marked tendency to deteriorate the LCoS performance in diffractive optics, some previous studies [13] have endeavored to totally report the modulator performance as a function of the electrical pulse scheme addressed to the device.

Other limitations in the performance of the LCoS device include phase modulation depths smaller than $2 \pi$, phase modulations not being linear with the applied voltage or available phases being limited to certain quantized values. The minimum Euclidean principle [14]-[16] is a method in which each complex value is projected over the closest available complex point in the modulation domain. This process enables most of the previously stated LCoS limitations to be solved and provides the best efficiency for our device. Its usefulness has been proved both in LCD [17] and in LCoS devices [13]. In the latter case the minimum Euclidean principle was applied in the presence of temporal phase fluctuations in the LCoS.

In LCD devices it was found that the phase modulation depth 
exhibited by the LCD strongly depends on the orientation and spatial frequency components of the displayed image [18]. Specifically the phase modulation depth depended on the magnitude of the local spatial frequency component along the horizontal direction. Along the vertical direction, the phase modulation depth did not depend on the spatial frequency. The phenomenon is related with the electronics driving the device and in no way related to liquid crystal physics [19]. It causes a reduction of the optical efficiency of a diffractive optical element (DOE) displayed onto this type of modulator. This anamorphic and spatial frequency dependent behavior, observed with phase DOEs in Ref. [18], had also been reported by other authors when using the LCD in the amplitude-only regime [20]-[23]. In general, the goal of these authors was to calculate the Modulation Transfer Function (MTF) of the LCD in order to characterize the device for optical processing [21]-[23] and for holographic data storage (see page 247 in Ref. [20]). In Ref. [18] it was developed an encoding algorithm based on the minimum Euclidean projection to display the diffractive element with an optimal modulation diffraction efficiency taking into account the anamorphic and spatial frequency dependent phenomenon. Specifically the algorithm was used to display Fresnel lenses onto the LCD. In principle, the anamorphic and spatial frequency dependent phenomenon has not been studied in LCoS devices. From the experience gathered with LCDs, it is clear that this phenomenon may significantly influence the performance of LCoS in a wide range of applications, thus in this paper we undertake the analysis of the phenomenon in LCOS devices.

The outline of this paper is as follows. In Section 2, we present the basic theory and the methodology employed in order to determine the anamorphic and spatial frequency dependent phenomenon. Section 3 provides the analysis of the results obtained when generating binary gratings. A comparative study between diffractive elements containing different frequencies and orientations has been conducted. Finally, Section 4 deals with the main conclusions derived from this study.

\section{LCOS DISPLAY ANAMORPHIC PHENOMENOM}

The purpose of this section is to briefly show the anamorphic and spatial frequency dependent phenomenon observed when diffractive gratings in different directions are displayed within the PA LCoS display.

This study has been undertaken for a $633 \mathrm{~nm}$ laser light source and for quasi-normal incidence. The light modulator used is a PA LCoS display distributed by HoloEye. The modulator works by reflection, therefore enabling a higher dynamic modulation range when compared to previous transmissive LCDs because of the double pass of the light through the device. It also presents a high resolution due to its $1920 \times 1080$ pixel screen; a high fill factor of $87 \%$ and a pixel size smaller than $8 \mu \mathrm{m}$. Voltages can be addressed to the LCoS by means of different electrical sequences also provided by HoloEye. In this article, we study the anamorphism for a sequence optimized at $633 \mathrm{~nm}$ wavelength light. We use the one providing the best trade-off between global retardance and tempo-

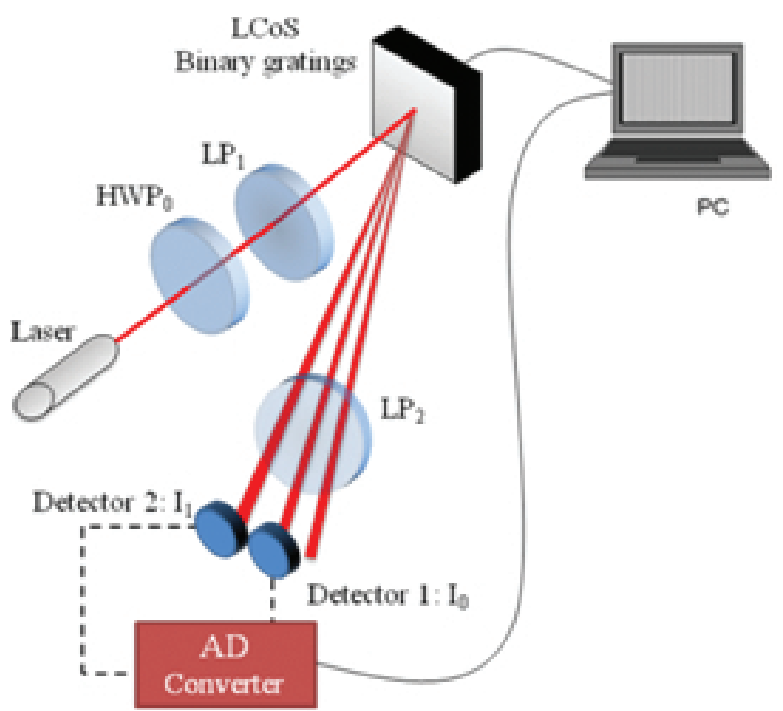

FIG. 1 Optical setup to capture the zero and first order intensities. $\mathrm{HWP}_{0}$ is a half-wave plate and LP are linear polarizers.

ral phase-fluctuations and thus resulting in the best available diffractive efficiencies.

The experimental methodology to determine the significance of the anamorphic and spatial frequency dependent phenomenon is similar to those proposed in Ref. [24, 25], where a binary grating is addressed to the display and the ratio between the first and zero diffraction orders is measured. This ratio depends on the phase and amplitude difference between the two levels of the grating. Since this is a parallel aligned LC device, it acts as a pure phase modulator when the input polarization is selected linear parallel to the LC director, and the phase modulation can be directly derived from the ratio of the intensities in the diffraction orders. In our case several binary gratings in the modulator are generated, each one having a different period and for the two Cartesian orientations: the horizontal or the vertical direction, (the period is respectively specified in rows/period and columns/period). As sketched in Figure 1, the LCoS display is impinged with an unexpanded $\mathrm{He}-\mathrm{Ne}$ laser beam at quasi-normal incidence $\left(2^{\circ}\right)$. Light coming from the laser goes through a half wave-plate $\left(\mathrm{HWP}_{0}\right)$ which enables the light intensity to be controlled. A polarizer is placed at the entrance of the incident beam, while another polarizer (set in the same position) is set at the exit of the beam reflected by the LCoS display. When the proper incident state of polarization is generated, the display works with a phaseonly modulation regime and depolarization effects can be removed provided that the beam is linearly polarized parallel to the LC director. After the last polarizer, two photodetectors are placed in a far diffraction plane, gathering both the zero and the first diffraction order intensities in synchronization. Finally, an AD converter allows the intensity values to be visualized on the PC screen. Analytical expressions for the intensity of the zero and first orders can be derived as a function of the phase difference, $\Phi$ :

$$
\begin{aligned}
I_{0}(t) & =\frac{1}{2}(1+\cos \Phi(t)), \\
I_{ \pm 1}(t) & =\frac{2}{\pi^{2}}(1-\cos \Phi(t)) .
\end{aligned}
$$


We note that we fix one of the levels in the binary grating to gray level zero, which shows no temporal phase fluctuations, whereas the other gray level is varied. Thus we measure the phase difference, $\Phi$, relative to the phase value associated to gray level zero. Once intensities have been measured, the instantaneous phase modulation can be found as follows:

$$
\cos (\Phi(t))=\frac{4 I_{0}(t)-\pi^{2} I_{1}(t)}{4 I_{0}(t)+\pi^{2} I_{1}(t)} .
$$

This expression presents an ambiguity for angles close to $\pi$. This can be solved by applying continuity considerations with the values obtained in the vicinity. With this diffractive-based method, the phase difference curves, equal to the retardance value of the LC layer for each binary grating as a function of the gray level and as a function of time can be obtained. Experimental curves have been determined for gratings with periods of $16,8,4$ and 2 pixels and generated in both the vertical and the horizontal directions.

\section{ANALYSIS OF THE ANAMORPHIC PHENOMENON}

In this section, we present the experimental results obtained when applying the previous method for a given electrical sequence (5_5 linear 2pi) [13]. Figure 2(a) shows the retardance curves obtained when performing the experiment for binary horizontal gratings (diffraction orders on the vertical axis) with consecutive periods of 16, 8, 4 and 2 rows/pixel. Analogously, Figure 2(b) shows the phase retardances obtained for the same sequence and also the same periods, but for vertical gratings (columns/period) instead.

It is easily noticeable that in the first case, for all the four periods, values maintain themselves relatively close each other throughout the gray levels. Only the smallest period $(\mathrm{P}=2)$ shows a slight deviation from the gray level 200 onwards. As the graph shows, differences between retardance values for different periods become more evident as the gray level increases, in such a way that for the 250 gray level, the period $\mathrm{P}=16$ reaches a global retardance of $325^{\circ}$ while the period $\mathrm{P}=2$ reaches $277^{\circ}$. This means that, even in the worst situation where the diffractive element generated contains very different periods and requires very high gray levels being addressed to the device, provided that the element has the periodic structure in the horizontal direction, the maximum difference between retardance values will be less than $50^{\circ}$. Thus, if addressing DOEs with horizontal periodicities higher than 2, the measure of a single retardance curve (for a single period) is enough to describe the LCoS display phase response.

When looking at the retardance values for the vertical gratings plotted in Figure 2(b), the situation becomes different. For the period $P=16$, retardance values are quite similar to those obtained for the horizontal grating. The global retardance in this case reaches a maximum of $323^{\circ}$, therefore showing that the LCoS performance is good enough when generating diffractive elements containing periodic patterns of low spatial frequency. Nevertheless, for vertical gratings, the global retardances decrease as the spatial frequency of the grating increases. For the period $\mathrm{P}=8$, the global retardance difference

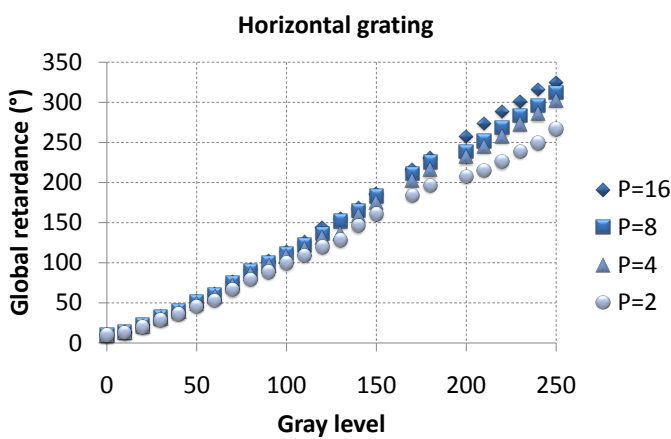

FIG. 2a Retardance values as a function of the gray level for horizontal gratings.

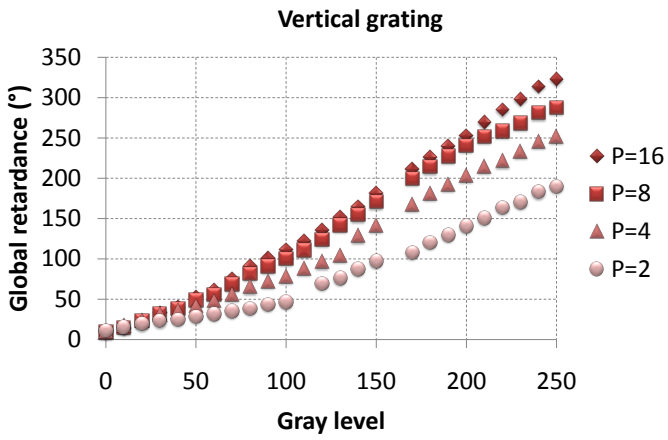

FIG. 2 b Retardance values as a function of the gray level for vertical gratings.

between the horizontal and the vertical grating at the 250 gray level is about $34^{\circ}$. This difference becomes $61^{\circ}$ for the period $\mathrm{P}=4$ and approximately $90^{\circ}$ when looking at the period $\mathrm{P}=2$. When working with vertical gratings (diffraction orders on the horizontal axis), the LCoS response varies substantially depending on the period of the grating generated. For the 250 gray level, the difference between the global retardance for the periods $\mathrm{P}=16$ and $\mathrm{P}=2$ is about $133^{\circ}$, while for horizontal gratings was of less than $50^{\circ}$.

After analyzing the results shown in Figure 2, it is clear that the anamorphic effect must be considered in order to properly control the LCoS display performance. For an appropriate generation of DOEs, the programmed phase distribution we desire to implement onto the LCoS display must be almost equal to the experimentally displayed one, allowing us to obtain the expected diffractive pattern. Thus, a reliable look-up table (LUT) is required. However, as a consequence of the anamorphic phenomenon, if we generate DOEs containing high frequency structures in the vertical direction, the use of a unique LUT (obtained by applying low frequency DOEs, for instance, binary gratings with a period higher than 8 pixels) is not sufficient. This is because into the high frequency range, the phase curves strongly vary with frequency (see Figure $2(b))$.

To solve this problem, a calibration for different LUTs related to each one of the involved periods is required in order to 


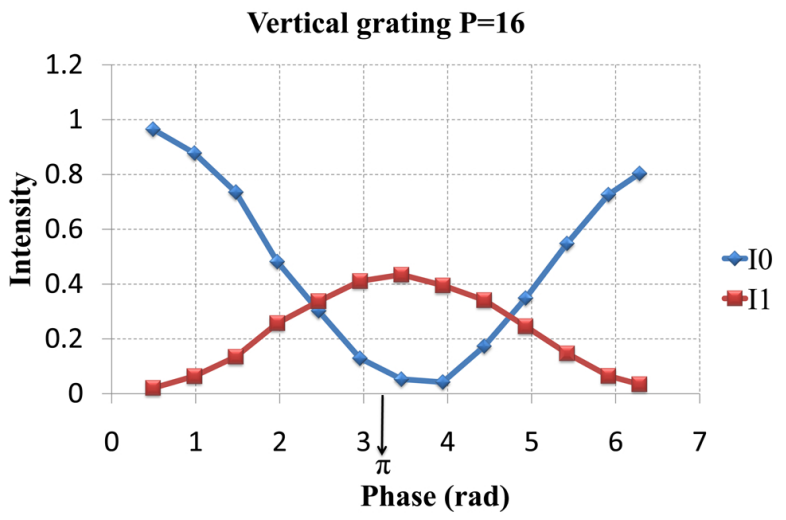

FIG. $3 a$

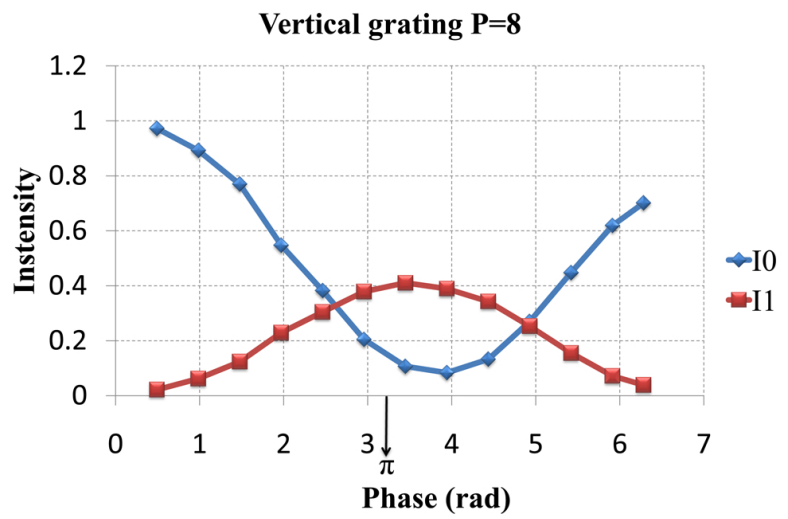

FIG. $3 b$

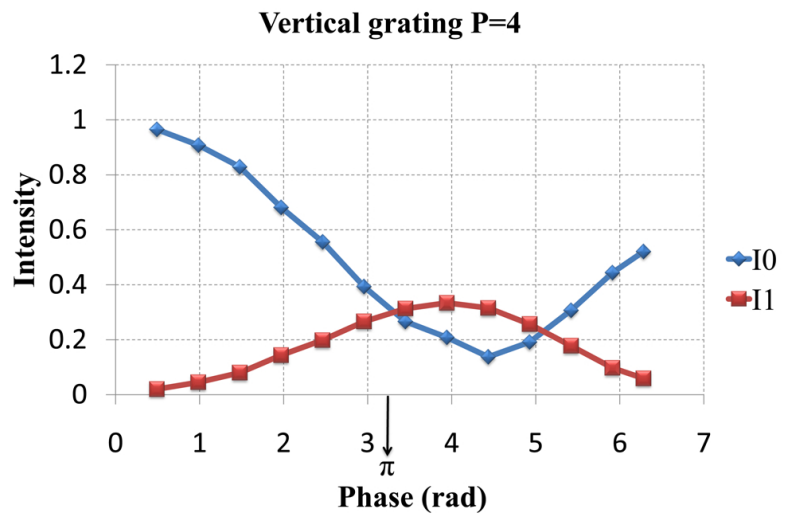

FIC. $3 C$

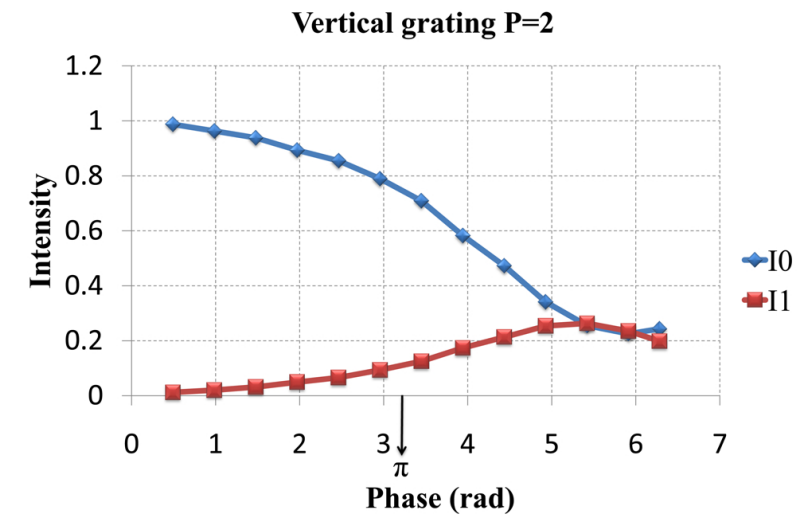

FIG. 3d Zero and first order diffraction intensities for a vertical binary grating.

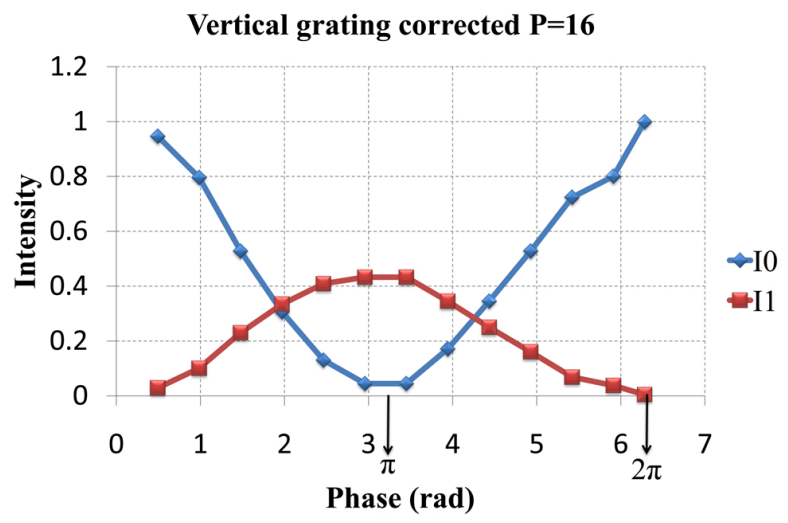

FIC. $3 a$

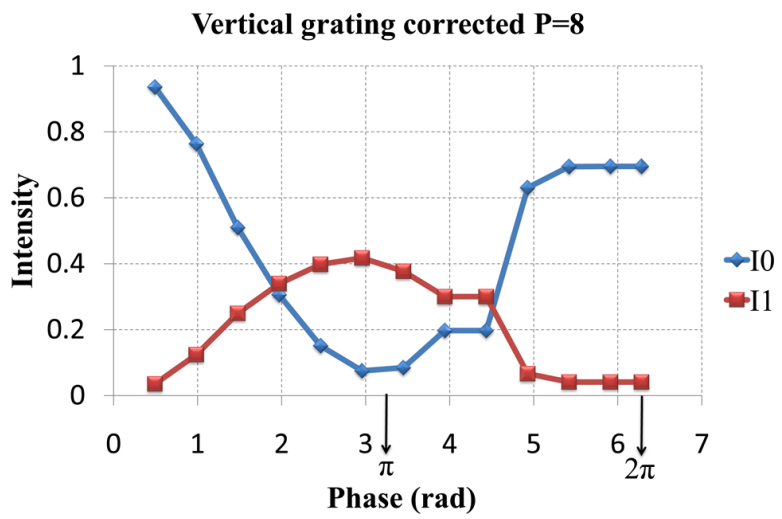

FIG. 3b

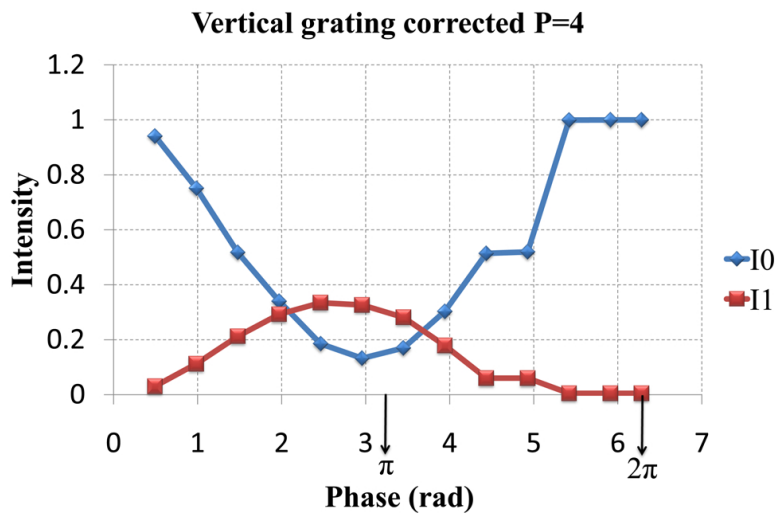

FIG. $3 \mathrm{C}$

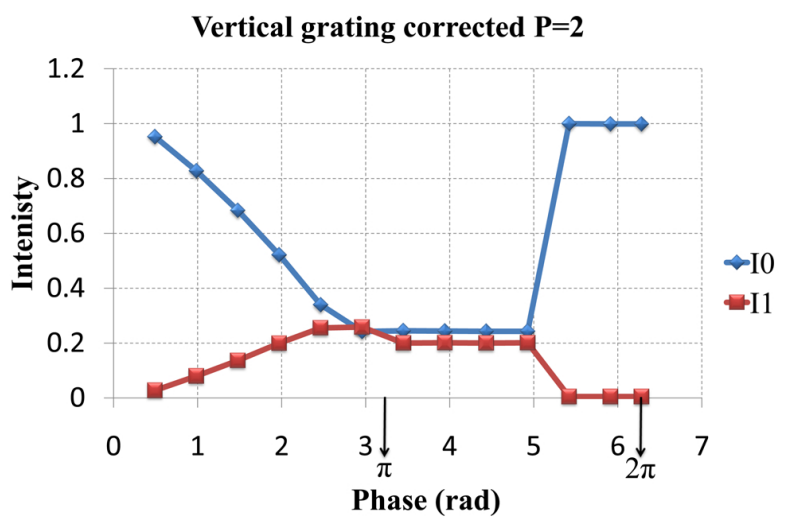

FIC. 3d 
achieve a finest relation between the desired phase distribution and the LCoS display performance. This correction can be performed with the corresponding LUT derived from the Minimum Euclidean Distance principle, assigning the closest available phase. Note that the correspondence between the programmed phase distribution and the LCoS display performance it is necessary not only when addressing binary DOEs but also for DOEs containing diverse gray levels or even continuous DOEs. In such cases, the application of the Minimum Euclidian Distance will be useful.

In order to provide a higher insight on this topic, we show different experimental results in Figure 3. On the one hand, Figure 3 (left-hand part) shows the zero and the first diffraction order intensities for the four different grating periods when applying a common look-up table and as a function of the desired phase difference values (i.e. the theoretical phase we want to implement). Note we use the LUT obtained when using binary gratings with a very low spatial frequency so that no anamorphic and frequency dependent behavior is present. On the other hand, in Figure 3 (right-hand part) we show the analogous results after applying the corresponding LUT specific for each spatial frequency. We call them the corrected gratings. Again, the x-axes represent the phase we want to address to the LCoS display.

The graphs on the left-hand, which correspond to the gratings not being corrected, show an agreement with Figure 2(b). When working with binary grating, first order maximums point out a phase value of $\pi$. Therefore, if the LCoS response were linear with the addressed voltage, these maximums should be placed at the $\pi$ value on the x-axis. However, the maxima are shifted substantially to the right as consecutive higher frequencies are considered, going far from the desired phase value. This is, as the grating period decreases, it is necessary to address higher voltages to the modulator in order to obtain the same phase values.

At this point, a correction can be performed with the corresponding LUT derived from the Minimum Euclidean Distance principle, assigning the closest available phase. The effect of the spatial frequency dependent LUT application can be seen on the right-hand part graphs. Intensity maxima are shifted closer to the $\pi$ value, thus making more linear the LCoS response with the addressed voltage. Therefore, as show the diffracted order maxima, by applying corrected LUTs we achieve phase differences closer to the expected ones. Once higher phase values are not available any more, the Euclidean method is applied. This can be observed because of the constant values set both at the maximum possible phase value and at the minimum value (the zero phase value is equivalent to $2 \pi)$. We note that since we are working with binary phase gratings, the maximum diffraction efficiency is limited to $40 \%$.

\section{CONCLUSION}

In this work, we study the anamorphic and spatial frequency dependent phenomenon of LCoS devices, which is related to the great dependence of the modulator response on the period and the spatial orientation of the diffractive element gen- erated. We have provided experimental evidence of this phenomenon, showing that diffractive elements containing periodic structures of high frequencies lead to a worst performance of LCoS displays. Furthermore, this effect is more obvious for horizontal periodic structures than for vertical ones because of the way in which the electrical pulses are addressed to the LCoS devices. This effect only appears for very high frequencies.

In order to solve this problem, spatial frequency dependent LUTs must be applied. Nevertheless, this model must be used taking into account the period and direction of the diffractive element generated. Experimental results show how corrected gratings provide more accurate response of the LCoS display.

\section{ACKNOWLEDGMENTS}

We acknowledge financial support from the Spanish Ministerio de Educación y Ciencia (grants FIS209-13955-C02-01 and FIS209-13955-C02-02) and from Generalitat de Catalunya (grant 2009 PIV 00051). C. Iemmi gratefully acknowledges the support of the Universidad de Buenos Aires and CONICET (Argentina).

\section{References}

[1] S. T. Wu, and D. K. Yang, Reflective Liquid Crystal Displays (John Wiley a Sons Inc., Chichester, 2005).

[2] J. Turunen, and F. Wyrowski, Diffractive Optics for Industrial and Commercial Applications (Akademie Verlag, Berlin, 1997).

[3] R. Dou, and M. K. Giles, "Closed-loop adaptive optics system with a liquid crystal television as a phase retarder" Opt. Lett. 20, 1583-1585 (1995).

[4] W. Osten, C. Kohler, and J. Liesener, "Evaluation and application of spatial light modulators for optical metrology" Opt. Pura Apl. 38, 71-81 (2005).

[5] A. De Martino, Y. K. Kim, E. Garcia-Caurel, B. Laude and B. Drévillon, "Optimized Mueller polarimeter with liquid crystal" 0pt. Lett. 28, 616-618 (2003).

[6] M. Anastasiadou, A. De Martino, D. Clement, F. Liège, B. LaudeBoulesteix, N. Quang, J. Dreyfuss, et. al., "Polarimetric imaging for the diagnosis of cervical cancer" Phys. Status Solidi 5, 5 (2008).

[7] A. Hermerschmidt, S. Osten, S. Krüger, and T. Blümel, “Wave front generation using a phase-only modulating liquid-crystalbased micro-display with HDTV resolution" Proc. SPIE 6584, 65840E (2007).

[8] J. R. Moore, N. Collings, W. A. Crossland, A. B. Davey, M. Evans, A. M. Jeziorska, M. Komarčević, et. al., "The silicon backplane design for an LCOS polarization-insensitive phase hologram SLM" IEEE Photonic Tech. L. 20, 60-62 (2008).

[9] A. Lizana, I. Moreno, A. Márquez, C. Iemmi, E. Fernández, J. Campos, and M. J. Yzuel, "Time fluctuations of the phase modulation in a liquid crystal on silicon display: characterization and effects in diffractive optics" Opt. Express 16, 16711-16722 (2008).

[10] A. Lizana, I. Moreno, A. Márquez, E. Also, C. Iemmi, J. Campos, and M. J. Yzuel, "Influence of the temporal fluctuations phenomena on the ECB LCOS performance" Proc. SPIE 7442, 74420G-1 (2009). 
[11] J. E. Wolfe, and R. A. Chipman, "Polarimetric characterization of liquid-crystal-on-silicon panels" Appl. Opt. 45, 1688-1703 (2006).

[12] A. Lizana, I. Moreno, C. Iemmi, A. Márquez, J. Campos, and M. J. Yzuel, "Time-resolved Mueller matrix analysis of a liquid crystal on silicon display" Appl. Opt. 47, 4267-4274 (2008).

[13] A. Lizana, A. Márquez, L. Lobato, Y. Rodange, I. Moreno, C. Iemmi, and J. Campos, "The minimum Euclidean distance principle applied to improve the modulation diffraction efficiency in digitally controlled spatial light modulators" Opt. Express 18, 10581-10593 (2010).

[14] I. Moreno, J. Campos, C. Gorecki, and M. J. Yzuel, "Effects of amplitude and phase mismatching errors in the generation of a kinoform for pattern recognition" Jpn. J. Appl. Phys. 34, 6423-6432 (1995).

[15] R. D. Juday, "Optical realizable filters and the minimum Euclidean distance principle" Appl. Opt. 32, 5100-5111 (1993).

[16] R. D. Juday, "Generality of matched filtering and minimum Euclidean distance projection for optical pattern recognition" J. Opt. Soc. Am. A 18, 1882-1896 (2001).

[17] I. Moreno, C. Iemmi, A. Márquez, J. Campos, and M. J. Yzuel,"Modulation light efficiency of diffractive lenses displayed onto a restricted phase-mostly modulation display" Appl. 0pt. 43, 6278-6284 (2004).

[18] A. Márquez, C. Iemmi, I. Moreno, J. Campos, and M. J. Yzuel, "Anamorphic and spatial frequency dependent phase modulation on liquid crystal displays. Optimization of the modulation diffraction efficiency" Opt. Express 13, 2111-2119 (2005).
[19] A. Márquez, I. Moreno, C. Iemmi, J. Campos, and M. J. Yzuel, "Electrical origin and compensation for two sources of degradation of the spatial frequency response exhibited by liquid crystal displays" Opt. Eng. 46, 114001, 1-11 (2007).

[20] H. J. Coufal, D. Psaltis, and B. T. Sincerbox, Holographic Data Storage (Springer-Verlag, Berlin, 2000).

[21] M. L. Hsieh, K. Y. Hsu, E. G. Paek, and C. L. Wilson, "Modulation transfer function of a liquid crystal spatial light modulator" Opt. Commun. 170, 221-227 (1999).

[22] M. L. Hsieh, E. G. Paek, C. L. Wilson, and K. Y. Hsu, “Performance enhancement of a joint transform correlator using the directionality of a spatial light modulator" Opt. Eng. 38, 2118-2121 (1999).

[23] P. Grother, and D. Casasent, "Modulation transfer function measurement method for electrically addressed spatial light modulators" Appl. Opt. 40, 5253-5259 (2001).

[24] Z. Zhang, G. Lu, and F. T. S. Yu, "Simple method for measuring phase modulation in liquid crystal televisions" Opt. Eng. 33, 3018-3022 (1994).

[25] J. Reményi, P. Vàrhegyi, L. Domján, P. Koppa, and E. Lörincz, "Amplitude, Phase, and Hybrid Ternary Modulation Modes of a Twisted-Nematic Liquid-Crystal Display at $\sim 400$ nm" Appl. 0pt. 42 3428-3434 (2003). 